\title{
Solar Flare Prediction and Magnetic Field Evolution in Solar Active Region
}

\author{
H. N. Wang, G. Q. Zhang, C. L. Zhu and J. L. Sun \\ Beijing Astronomical Observatory, CAS, Beijing 100012, China and \\ National Astronomical Observatories, CAS, Beijing 100012, China
}

\begin{abstract}
The authors propose a number of empirical criteria for prediction of solar flares based on many years of observations at Huairou Solar Observing Station of Beijing Astronomical Observatory.
\end{abstract}

\section{Introduction}

The energy of solar flares is released from magnetic fields in solar active regions. Till now, theoretical studies have not revealed completely how this energy is released from solar magnetic fields. Therefore, solar flare prediction is still based on a statistical relationship between solar flares and magnetic evolution. In order to establish this relationship more firmly, sunspot groups are classified according to their morphology and magnetic configuration. However, these classifications provide limited information on solar active regions.

Solar magnetic fields have been measured for long. Evolution of Solar magnetic field can be studied not only morphologically, but also quantitatively. Many solar observers have their own experiences for solar flare prediction, since they observed a lot of pre-flare phenomena. Based on long-term solar magnetic field observation at Huairou Solar Observing Station of Beijing Astronomical Observatory, we propose the following empirical criteria for solar flare prediction.

\section{New Criteria}

The following new criteria for solar flare predictions are proposed:

1. Large horizontal gradient of longitudinal magnetic field.

2. Intensity of magnetic field increase rapidly.

3. Abnormal polarity distribution in f-p sunspot pairs.

4. Newly emerging magnetic flux.

5. Magnetic flux squeezing into opposite polarity region.

6. Extremely long polarity reversal line.

7. Merging magnetic flux in areas with the same polarities. 
8. Large tilt angle of magnetic axis in an active region.

These criteria have been used at our observatory for the past 10 years. More than sixty percent of all important solar flares ( $>M$ class) has been correctly predicted within 48 hours.

\section{Discussion}

(1) Transverse magnetic fields can also be used to define new criteria;

(2) Since we have developed a new numerical method for 3-D magnetic field reconstruction, evolution of 3-D magnetic fields in solar active regions can be analyzed and a new prediction model will be proposed. 